\title{
Automatic Segmentation and Classification of Masses from Digital Mammograms
}

\author{
${ }^{1}$ Basma A. Mohamed, ${ }^{1}$ Nancy M. Salem, ${ }^{1}$ Marwa M. Hadhoud and ${ }^{1,2}$ Ahmed F. Seddik \\ ${ }^{1}$ Faculty of Engineering, Biomedical Engineering Department, Helwan University, Cairo, Egypt; \\ ${ }^{2}$ Faculty of Computer Science, Nahda University (NUB) Egypt \\ eng_basma1990@yahoo.com; nancy_salem@h-eng.helwan.edu.eg; \\ marwa_hadhoud@h-eng.helwan.edu.eg; ahmed_sadik@h-eng.helwan.edu.eg
}

\begin{abstract}
Breast cancer is one of the leading causes of death among female cancer patients. Mammography is the most efficient method for the early detection of abnormalities that are associated with breast cancer. Masses and micro calcifications are the most popular abnormalities that indicate breast cancer. The proposed paper intends to develop an automated system for assisting the analysis of digital mammograms. First, a preprocessing step is applied to enhance images followed by a segmentation step that is based on morphological operations and Otsu's thresholding techniques. Thereafter, shape features are extracted from the segmented region and used in the classification process. Finally, the classification step to classify the segmented shape as round, oval, lobular, or irregular. The algorithm is tested using $\mathbf{2 7 0}$ mammogram images from the Women Health Care Program (WHC) and 142 publicly available images from the Digital Database for Screening Mammography (DDSM). Results show that the proposed technique effectively detects and segments masses from mammogram images. The shape of segmented masses is classified into either round, oval, lobular, or irregular. Round and oval shapes are classified with $100 \%$ accuracy while lobular and irregular shapes results in accuracy of $93 \%$ using the ANN for the WHC dataset and $100 \%$. On the other hand, accuracy for images from the DDSM is $100 \%$ and $91.3 \%$ respectively.
\end{abstract}

Keywords: Breast Cancer, Digital Mammograms, Otsu's threshold, BI-RADS ${ }^{\text {TM }}$ Categories.

\section{Introduction}

Breast cancer is the most commonly detected type of cancer among women all over the world. Due to the unawareness, there is an increase in the cases that are detected and diagnosed in advanced stages of the cancer [1]. To help in the early detection of this type of cancer, screening programs are required to detect the early signs of the breast cancer. Currently, mammography is a very effective method for the early detection of masses or abnormalities. It can detect 85 to 90 percent of all breast cancers. Masses and micro calcifications are signs that indicate breast cancer.

According to Breast Imaging Reporting and Data System (BIRADS), the shape, size, margins, and density are the features that are used to characterize masses [2-4]. The shape could be round, oval, lobular, or irregular. The shape and margin properties play an important role in classifying the masses. It is observed that benign tumors have a round, oval or lobular shapes while malignant have irregular or lobular shapes with a speculated margin. With the advances in computer technology, Computeraided detection systems (CAD) have been developed to improve breast cancer screening and 
Basma A. Mohamed, Nancy M. Salem, Marwa M. Hadhoud and Ahmed F. Seddik; Automatic Segmentation and Classification of Masses from Digital Mammograms. Advances in Image and Video Processing, Volume 4 No 4, August (2016); pp: 17-23

diagnosis. The CAD system helps in processing large number of images and it involves digital image processing, image analysis, artificial intelligence, and software development.

In the literature, many methods have been developed for the detection and classification of masses or microcalcifications [5-13]. For the detection of masses, the Growing Neural Gas (GNG) has been used with the Supported Vector Machine (SVM) [5]. A sensitivity of $89.3 \%$ is reported when using a set of 997 images from the DDSM database. In the work proposed by Tzikopoulosa et al. [6], the breast density estimation and the asymmetry detection are used for the segmentation and classification of mammograms from the mini-MIAS database. Statistical features for pair of mammograms were computed and the difference is used to define the asymmetry followed by SVM with a success rate of $84.47 \%$.

Region growing and Wavelet Transform are used in the work proposed in [7]. Features are extracted and the classification is performed using the SVM. Images were used from the MIAS database and a local dataset from the hospital of Istanbul University (I.U.) in Turkey. The K-means clustering method is used in [8] to detect masses. A set of 650 images from the DDSM database is used and results in a sensitivity of $83.24 \%$, and a specificity of $84.14 \%$. The Artificial Neural Network (ANN) is used with shape and texture features in [9]. A set of 226 images (109 malignant and 117 benign masses) were used. The sensitivity and specificity are $78.1 \%$ and $79.1 \%$ respectively.

Regarding the shape of the masses, Vadivel and Surendiran $[10,11]$ developed an algorithm for classifying masses into the four basic shapes; round, oval, lobular, and irregular. Shape features have been extracted and then the feature vector is applied to the C5.0 decision tree algorithm and fuzzy inference systems. The algorithm is tested using 224 mammogram masses from DDSM database. They achieved accuracy of $87.76 \%$ in differentiating the four mass shapes, $100 \%$ in differentiating between round and oval masses, and accuracy $93.29 \%$ in differentiating between lobular and irregular masses [11].

The SVM and the Linear Discriminant Analysis (LDA) have been used to classify 200 and 3600 regions of interest from the MIAS and DDSM databases respectively. Results from the two classifiers are compared and results show a better performance for the SVM as reported by Costa et al. [12].

In this paper, a new method to segment and classify masses from digital mammograms is proposed. This paper is organized as follows: Section 2 presents the proposed detection and classification method. Section 3 describes the experimental results and the conclusion is given in Section 4.

\section{Proposed Method}

This section describes the proposed method for detection and classification of mases from digital mammograms. Figure 1 shows a block diagram of the proposed method and its four main stages. In the beginning, a preprocessing is applied to enhance images followed by a segmentation stage that is based on morphological operations and Otsu's thresholding techniques to detect masses. Thereafter, shape and texture features are extracted from the segmented region. Finally, the classification stage which consists of two sequential steps; the first one to distinguish masses as either regular or irregular. The second step is applied to distinguish the regular mass as either oval or round and the irregular mass as either lobular or irregular. Three different classifiers have been used in the classification stage which are: the ANN, the SVM and the K-Nearest Neighbor classifier (KNN). 


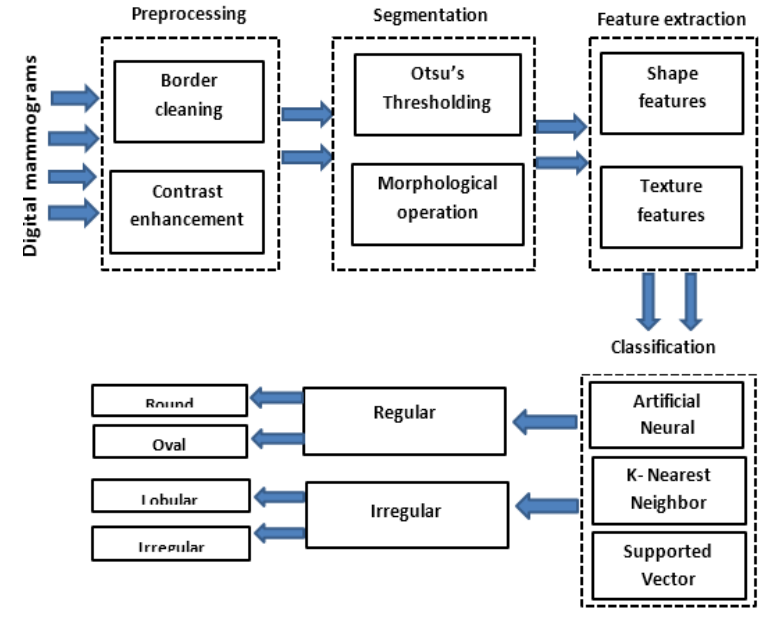

Figure 1: Block diagram of the proposed method.

\subsection{Datasets}

In this study, two different databases are used. 270 images have been collected from Women Health Care Program (WHC) [14], which is a large-scale initiative for women's healthcare comprising digital mammograms for early detection of breast cancer. These images were previously investigated and labelled by expert radiologists based on technical experience and biopsy. The database contains 270 mammograms including 185 malignant and 85 benign cases (73 are round, 83 are oval, 18 are lobular and 96 are irregular). All images are digitized at a resolution of 2294×1914 pixels. 142 mammograms have also been used from the DDSM digital image database [15] that contains 96 malignant and 46 benign masses ( 7 are round, 17 are oval, 21 are lobular and 96 are irregular).

\subsection{Pre-processing}

A pre-processing stage is required to improve the image quality and to enhance the segmentation results. Image border cleaning is applied to remove any artifacts like the pectoral muscle and any labels attached to the border of the image. Then the intensity adjustment is applied to improve the contrast between the mass structures and surrounding texture of the breast tissues. These steps are shown in Figure 2.

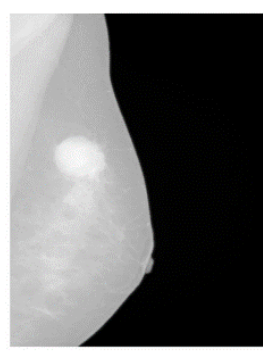

(a)

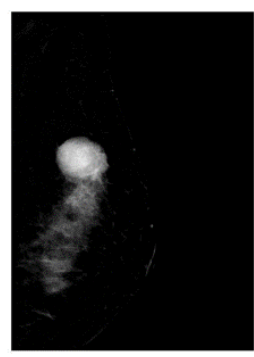

(b)

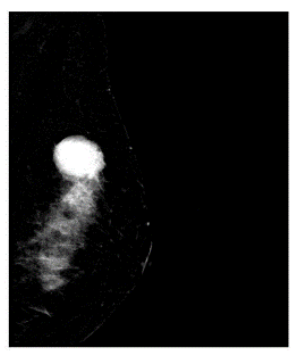

(c)

Figure 2: The preprocessing stage: (a) Original mammogram, (b) Border clearing, and (c) Contrast adjustment.

\subsection{Mass Segmentation}

The goal of segmentation stage is to identify the region of interest (mass) and this is achieved by applying the Otsu's threshold [16] and morphological operations [17] such as erosion and opening to extract this region. In this paper, the Otsu's threshold technique alone is not efficient enough to get a good result in identifying the mass region, so a constant is added to a global threshold that can be 

Classification of Masses from Digital Mammograms. Advances in Image and Video Processing, Volume 4 No 4, August (2016); pp: 17-23

used to convert an intensity image to a binary image. The constant value lies between 0 and 1 . Figure 3 shows segmented masses.
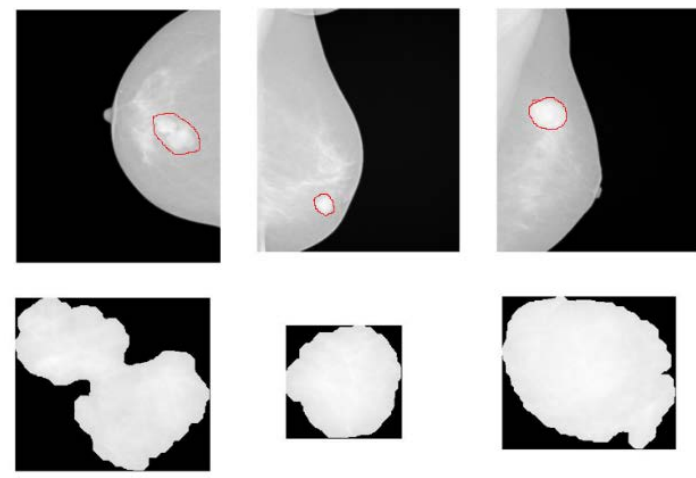

Figure 3: Examples of extracted masses.

\subsection{Feature Extraction}

Feature extraction is very important in the classification of masses. There are many different features that are used in mass classification; the most common features are texture and shape features. The BIRADS specifies that benign masses are round, oval, lobular and malignant masses are lobular or irregular shape. In this work, shape and texture features such as area, perimeter, major axis, minor axis, thinness ration, eccentricity, equivdiameter, dispersion, compactness, circularity, roundness, elongation, shape index and entropy. These features are extracted from segmented regions and then the features are normalized and ranked using absolute value two-sample t-test to select the most effective features.

\subsection{Classification}

In this stage, the segmented mass is firstly classified as either regular or irregular; then the regular masses are classified into round or oval and the irregular masses into lobular or irregular. In this paper, a comparison is performed between ANN [18], K-Nearest Neighbor [19] and SVM.

\section{Experiments and Results}

The MATLAB 2015 software is used to implement the proposed algorithm. In the beginning, the pectoral muscle removal and image enhancement were applied to improve the mass segmentation. As mammograms have different intensity contrast levels as a result of various breast density levels for different patients, it is difficult to select an optimal image enhancement technique. Masses were brighter than the surrounding area, so it can be detected and segmented by thresholding. The Otsu threshold is used to obtain a binary image followed by morphological operators to remove isolated pixels that are not related to the mass. Experiments show that results of the mass detection improved when adding a predefined constant the threshold value obtained by the Otsu threshold algorithm.

For images from the WHC, the segmentation results in masses detection with $100 \%$ accuracy. For images from the DDSM, accuracy of $90 \%$ is obtained.

Segmented masses are used to extract 15 shape and margin features. These features are extracted from segmented regions and then features are normalized and ranked using absolute value twosample t-test to know the most significant features. The features are ranked as follows: 


\begin{tabular}{|c|c|}
\hline Thinness ratio & Major axes /minor axes \\
\hline Compactness & Perimeter \\
\hline Elongation & Area/perimeter \\
\hline Shape index & Major axes \\
\hline Circularity 1 & Dispersion \\
\hline Entropy & Equiv diameter \\
\hline Eccentricity & Area \\
\hline & Minor axes \\
\hline
\end{tabular}

The classification stage consists of two steps; first step is to classify masses as either regular or irregular. Experimental results for classifying the masses as either regular or irregular of the first and second databases are shown in Figure 4. Different numbers of features have been used with three different classifiers. As shown in Figure 4-a for the WHC dataset, when using the thinness ratio only, a result of $100 \%$ is obtained with the three classifiers. Best results obtained when using the ANN with less number of features and then decreased with increasing the number of features. Second best results obtained when using the SVM classifier. The KNN has better performance with two to four features only.

As shown in Figure 4-b for mammograms from the DDSM dataset, best results obtained when using a feature vector of seven to 11 feature with the ANN or the SVM. Results using the KNN is about $93 \%$.

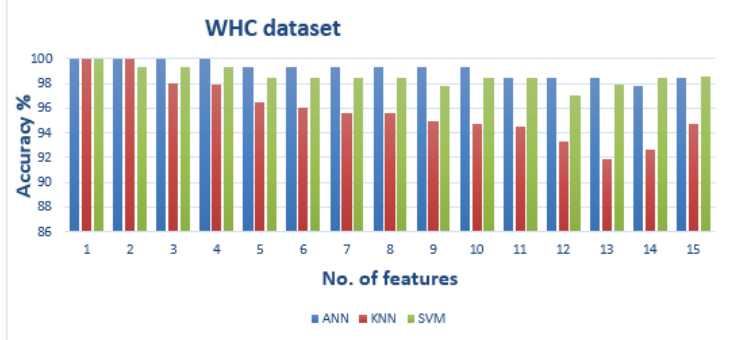

(a)

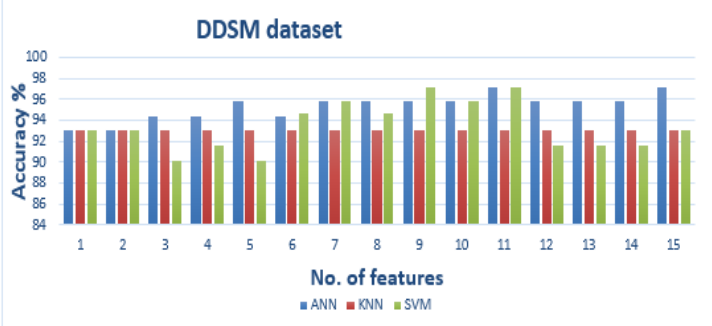

(b)

Figure 4: First classification step using: (a) WHC dataset, and (b) DDSM dataset

The second step of the classification is used to classify regular masses into round or oval and irregular masses into lobular or irregular. Results of this step are summarized in Table 1 when one feature (roundness) only is used. A comparison between the proposed method and other methods from the literature is summarized in Table 2.

Results are compared to these reported in $[10,11]$. The proposed algorithm used less number of features for the first classification step. It is important to declare that a very good performance is obtained when using thickness ratio as one feature in the first step with the ANN, SVM and KNN with the WHC dataset. A set of seven to eleven features were enough to get good performance with the DDSM dataset. On the other hand, there is only one feature is used in the second step of classification

Table 1: Performance of the second classification step.

\begin{tabular}{|c|c|c|c|c|}
\hline \multirow{2}{*}{ Dataset } & \multirow{2}{*}{ Shape } & \multicolumn{3}{|c|}{ Accuracy \% } \\
\hline & & ANN & KNN & SVM \\
\hline \multirow[b]{2}{*}{ WHC dataset } & Round vs. oval & $100 \%$ & $100 \%$ & $98.7 \%$ \\
\hline & $\begin{array}{l}\text { Lobular vs. } \\
\text { irregular }\end{array}$ & $93 \%$ & $92 \%$ & $84 \%$ \\
\hline \multirow[b]{2}{*}{ DDSM dataset } & Round vs. oval & $100 \%$ & $100 \%$ & $92.9 \%$ \\
\hline & $\begin{array}{l}\text { Lobular vs. } \\
\text { irregular }\end{array}$ & $91.3 \%$ & $90 \%$ & $85 \%$ \\
\hline
\end{tabular}


Table 2: Performance comparison

\begin{tabular}{|c|c|c|c|c|c|}
\hline \multirow{2}{*}{ Methods } & \multirow{2}{*}{ Database } & \multirow{2}{*}{$\begin{array}{c}\text { No. of } \\
\text { Features }\end{array}$} & \multirow{2}{*}{ Classifier } & \multicolumn{2}{|c|}{ Accuracy } \\
\hline & & & & RO & IL \\
\hline Surendiran [10] & DDSM & 19 & ANN & $98.32 \%$ & NA \\
\hline Vadivel [11] & DDSM & 17 & $\begin{array}{c}\text { Fuzzy inference } \\
\text { system }\end{array}$ & $100 \%$ & $93.29 \%$ \\
\hline \multirow{6}{*}{ Proposed method } & \multirow{3}{*}{$\begin{array}{c}\text { WHC Local } \\
\text { dataset }\end{array}$} & \multirow{3}{*}{15} & ANN & $100 \%$ & $93 \%$ \\
\hline & & & KNN & $100 \%$ & $92 \%$ \\
\hline & & & SVM & $98.7 \%$ & $84 \%$ \\
\hline & \multirow{3}{*}{ DDSM } & \multirow{3}{*}{15} & ANN & $100 \%$ & $91.3 \%$ \\
\hline & & & KNN & $100 \%$ & $90 \%$ \\
\hline & & & SVM & $92.9 \%$ & $85 \%$ \\
\hline
\end{tabular}

\section{Conclusion}

In this paper, an algorithm for automated detection and classification of masses in digital mammograms is proposed. The method consists of four main stages, preprocessing, segmentation, feature extraction, and classification. The proposed algorithm is tested using a set of 270 digital mammogram images from the WHC and 142 mammograms from the DDSM digital image database. Results show that the proposed technique effectively detects and segments all masses from the mammogram images with accuracy of $100 \%$ and $90 \%$ for the WHC and DDSM datasets respectively. The classification stage consists of two steps. Comparable results were obtained when using the ANN classifier to other methods in the literature. In this paper, three different classifiers were tested and results show a good performance for the ANN compared to the KNN and SVM.

\section{REFERENCES}

[1] Breast Cancer Foundation of Egypt (BCFE), http://www.bcfe.org/en/index.php, 2014.

[2] Verma, B., P. McLeod, and A. Klevansky, A novel soft cluster neural network for the classification of suspicious areas in digital mammograms, Pattern Recognition, 2009. 42 (9): p. $1845-1852$.

[3] Heath, M., et al., The digital database for screening mammography, Proceedings of the International Workshop on Digital Mammography 2000. p. 212-218.

[4] ACR, Breast imaging reporting and data system (BI-RADS), Breast Imaging Atlas, 4th ed., American College of Radiology, Reston, VA, 2010.

[5] Oliveira Martins, L., et al., Detection of breast masses in mammogram images using growing neural gas algorithm and Ripley's K function, Journal of Signal Processing Systems, 2009. 55 $(1-3)$ : p. 77-90. 
[6] Tzikopoulosa, S., et al., A fully automated scheme for mammographic segmentation and classification based on breast density and asymmetry, Computers Methods and Programs in Biomedicine, 2011. (102): p. 47-63.

[7] Görgel, P., A. Sertbas, and O. Ucan, Mammographical mass detection, and classification using Local Seed Region Growing- Spherical Wavelet Transform (L SRG- SWT) hybrid scheme, Computers in Biology and Medicine, 2013. 43: p. 765-774.

[8] Nunes, A., A. Silva, and A. Paiva, Detection of masses in mammographic images using geometry, Simpson's Diversity Index and SVM, Journal of Signal and Imaging Systems Engineering, 2010. 3(1): p. 43-51.

[9] Retico, A., et al., An automatic system to discriminate malignant from benign massive lesions on mammograms, Nuclear Instrumentation and Methods in Physics Research, 2006. 569(2): p. 596-600.

[10] B. Surendiran and A. Vadivel, Classifying mammographic masses into BI-RADSTM shape categories using various geometric and shape features, International Journal of Biomedical Signal Processing, 2011. 2(1): p. 43-47.

[11] Vadivel, A., and B. Surendiran, A fuzzy rule-based approach for characterization of mammogram masses into BI-RADS shape categories, Computers in Biology and Medicine, 2013. 43: p. 259-267.

[12] Costa, D., et al., Independent component analysis in breast tissues mammograms images classification using LDA and SVM, Information technology Application in Biomedicine, 2007. p. 231-234.

[13] De Nazar Silva, J., et al., Automatic detection of masses in mammograms using quality threshold clustering, correlogram function, and SVM, Journal of Digital Imaging, 2015. 28 (3): p. 323-373.

[14] Women Health Care Program, http://www.whop.gov.eg, 2007.

[15] Heath, M., K. Bowyer, and D. Kopans, Current status of the digital database for screening mammography, Digital Mammography, Kluwer Academic Publishers: p. 457-460.

[16] Otsu, N., A threshold selection method from gray-level histograms, Systems, Man, and Cybernetics, IEEE Transactions on, 1979. 9(1): p. 62-66.

[17] Gonzalez, R., R. Woods, and S. Eddins, Digital image processing using MATLAB, Gatesmark Publishing; 2nd edition, 2009.

[18] Schalkoff, R., Artificial neural networks. McGraw Hill, Publishers, 1997. p. 146-118.

[19] Altman, N. S., An introduction to kernel and nearest-neighbor nonparametric regression. The American Statistician, 1992. 46(3): p. 175-185. 